hep-th/0002212, CINVESTAV-FIS-00/08

\title{
Deformation Quantization of Bosonic Strings
}

\author{
H. García-Compeán, ${ }^{a}$ 国 J.F. Plebański, ${ }^{a}{ }^{2}$ M. Przanowski ${ }^{b}, a$ 国 and F.J. Turrubiates ${ }^{a}{ }^{a}$ \\ ${ }^{a}$ Departamento de Física \\ Centro de Investigación y de Estudios Avanzados del IPN \\ Apdo. Postal 14-740, 07000, México D.F., México \\ ${ }^{b}$ Institute of Physics \\ Technical University of Łódź \\ Wólczańska 219, 93-005, Eódź, Poland
}

\begin{abstract}
Deformation quantization of bosonic strings is considered. We show that the light-cone gauge is the most convenient classical description to perform the quantization of bosonic strings in the deformation quantization formalism. Similar to the field theory case, the oscillator variables greatly facilitates the analysis. The mass spectrum, propagators and the Virasoro algebra are finally described within this deformation quantization scheme.
\end{abstract}

February, 2000

\footnotetext{
1 compean@fis.cinvestav.mx

2 pleban@fis.cinvestav.mx

3 przan@fis.cinvestav.mx

4 fturrub@fis.cinvestav.mx
} 


\section{Introduction}

String theory is one of the most beautiful attempts to reconcile quantum mechanics and general relativity (for a review see [1, 2, 3, 3, 4]). From the physical point of view it is our best understanding of all matter and their interactions into an unified scheme. On the mathematical side, string theory has been used to motivate unsuspected interplay among some mathematical subjects. At the perturbative level, it is well known that string theory is related to the theory of Riemann surfaces [5] and some aspects of algebraic geometry and Mirror symmetry (see for instance [6]).

Non-perturbative revolution of string theory, through the introduction of D-branes and duality, have shown to be related to some aspects of toric geometry (see for instance [7], K-theory (see the Witten seminal paper, [8]), noncommutative geometry and deformation quantization theory [9, 10, 11]. This latter is relevant for the description of the low energy effective theory of open strings on the D-brane world-volume, when a non-zero NeveuSchwarz $B$-field is introduced. Thus, in this context, deformation quantization describes properly the noncommutative space time instead of the standard quantization of the phase space of the two-dimensional conformal field theory.

However, it would be important to describe the noncommutativity of space time and the quantization within the same framework of deformation quantization theory. In order to do that one has to quantize first string theory within this framework. This is something which has not been done yet in the literature. And at the same time this is the natural extension of the deformation quantization of classical fields developed in [12-18].

The purpose of the present paper is to give the first step into this unified description of quantum and noncommutativity in string theory. We will consider here the deformation quantization of the bosonic string theory. The fermionic case is leave for a forthcoming paper. In Sec. 2 we overview the preliminaries and notation of string theory in order to prepare the theory for quantization. Sec. 3 is devoted to do the quantization of bosonic strings by using deformation quantization theory. In Sec. 4 we describe the Casimir effect and the normal ordering within deformation quantization formalism. Correlation functions and Green functions for the bosonic string are computed in Sec. 5. Finally in Sec. 6 we give our final comments. 


\section{Classical Strings}

In this section we give a brief review of the classical bosonic string theory (for further details see [回,2,3, 团).

We consider the string world-sheet $\Sigma$ embedded into the $D$-dimensional space-time $M$ of Lorentzian metric $\eta_{\mu \nu}=\operatorname{diag}(-1,1, \ldots, 1), \mu, \nu=0,1, \ldots, D-1$. This embedding is defined by

$$
X^{\mu}=X^{\mu}\left(\sigma^{a}\right), \quad \mu=0,1, \ldots, D-1 \text { and } a=0,1
$$

where $X^{\mu}$ are the space-time coordinates and $\sigma^{a}$ stand for the coordinates on the worldsheet $\Sigma$. (In what follows the Greek indices $\mu, \nu, \ldots=0,1, \ldots, D-1$ correspond to the space-time components, the Latin indices $a, b, \ldots=0,1$ refer to the world-sheet coordinates).

Let $g_{a b}$ be a Riemannian metric of the Lorentzian signature $(-,+)$ on $\Sigma$. To find the equations of motion for the string we use the Polyakov action

$$
S_{P}=S_{P}\left[X^{\mu}, g_{a b}\right]=-\frac{T}{2} \int_{\Sigma} d^{2} \sigma \sqrt{-g} g^{a b} \partial_{a} X^{\mu} \partial_{b} X_{\mu}
$$

where $T$ denotes the string tension. Then the equations of motion read

$$
\frac{\delta S_{P}}{\delta X^{\mu}}=0 \Longleftrightarrow \partial_{a}\left(\sqrt{-g} g^{a b} \partial_{b} X^{\mu}\right)=0
$$

and

$$
\frac{\delta S_{P}}{\delta g^{a b}}=0 \Longleftrightarrow T_{a b}:=-\frac{2}{\sqrt{-g}} \frac{\delta S_{P}}{\delta g^{a b}}=T\left(\partial_{a} X^{\mu} \partial_{b} X_{\mu}-\frac{1}{2} g_{a b} g^{c d} \partial_{c} X^{\mu} \partial_{d} X_{\mu}\right)=0 .
$$

Of course, Eqs. (2.3) are the Laplace-Beltrami equations for $X^{\mu}$ on $\Sigma$, and Eqs. (2.4), saying that the energy-momentum tensor $T_{a b}$ vanishes, are the constraints of the string theory. One can quickly find that the constraint equations (2.4) give

$$
g_{a b}=\Omega^{2} \partial_{a} X^{\mu} \partial_{b} X_{\mu}
$$


with $\Omega=\Omega\left(\sigma^{a}\right)$ being an arbitrary nowhere vanishing function on $\Sigma$. The relation (2.5) means that the metric $g_{a b}$ is conformally equivalent to the induced metric $h_{a b}=\partial_{a} X^{\mu} \partial_{b} X_{\mu}$ on $\Sigma$. The form of the action (2.2) tells us that the theory is invariant with respect to: $(i)$ Poincaré transformations on $M,(i i)$ diffeomorphisms of $\Sigma$ and (iii) conformal rescalings of $g_{a b}$. The $(i i)$ and $(i i i)$ symmetries enable one to choose the coordinates $\sigma^{a}$ and the function $\Omega$ so that (conformal gauge)

$$
\begin{gathered}
\left(\sigma^{0}, \sigma^{1}\right) \equiv(\tau, \sigma), \quad 0 \leq \sigma \leq \pi, \\
\left(g_{a b}\right)=\left(\begin{array}{cc}
-1 & 0 \\
0 & 1
\end{array}\right) .
\end{gathered}
$$

With (2.6) assumed the equations of motion (2.3) take the simple form of the Klein-Gordon equations

$$
\ddot{X}^{\mu}-X^{\prime \prime \mu}=0
$$

where $\dot{X}^{\mu} \equiv \partial_{\tau} X^{\mu}$ and $X^{\prime \mu} \equiv \partial_{\sigma} X^{\mu}$.

Then the constraint equations (2.4) read

$$
\begin{gathered}
T_{01}=T_{10}=0 \Longleftrightarrow \dot{X}^{\mu} \dot{X}_{\mu}=0 \\
T_{00}=T_{11}=0 \Longleftrightarrow \dot{X}^{\mu} \dot{X}_{\mu}+X^{\prime \mu} X_{\mu}^{\prime}=0 .
\end{gathered}
$$

The Polyakov action (2.2) is now

$$
S_{P}=-\frac{T}{2} \int d \tau \int_{0}^{\pi} d \sigma\left(-\dot{X}^{\mu} \dot{X}_{\mu}+X^{\prime \mu} X_{\mu}^{\prime}\right) .
$$

In the case of the open string, i.e. $X^{\mu}(\tau, 0) \neq X^{\mu}(\tau, \pi)$ the action (2.9) leads to the equations (2.7) if the following boundary conditions are imposed for each $\tau$

$$
X^{\prime \mu}(\tau, 0)=0, \quad \text { and } \quad X^{\prime \mu}(\tau, \pi)=0
$$

For the closed string one has from the very definition 


$$
X^{\mu}(\tau, 0)=X^{\mu}(\tau, \pi)
$$

We will deal separately with the closed and open strings.

\subsection{Closed Strings}

The general solution of Eqs. (2.7) satisfying the boundary conditions (2.11) can be written in the form of the following series

$X^{\mu}(\tau, \sigma)=x^{\mu}+\frac{1}{\pi T} p^{\mu} \tau+\frac{1}{\sqrt{2 \pi T}} \sum_{n \neq 0} \sqrt{\frac{\hbar}{2|n|}}\left\{a_{n}^{\mu} \exp (2 i(n \sigma-|n| \tau))+a_{n}^{\mu *} \exp (-2 i(n \sigma-|n| \tau))\right\}$

where $x^{\mu}$ and $p^{\mu}$ are real and the star " $*$ " stands for the complex conjugation.

The conjugate momentum $\Pi^{\mu}$ of $X^{\mu}$ is as usual defined by

$$
\begin{gathered}
\Pi^{\mu}(\tau, \sigma)=\eta^{\mu \nu} \frac{\delta L}{\delta \dot{X}^{\mu}(\tau, \sigma)}=T \dot{X}^{\mu}(\tau, \sigma) \\
=\frac{1}{\pi} p^{\mu}+i \sqrt{\frac{T}{2 \pi}} \sum_{n \neq 0} \sqrt{2 \hbar|n|}\left\{a_{n}^{\mu *} \exp (-2 i(n \sigma-|n| \tau))-a_{n}^{\mu} \exp (2 i(n \sigma-|n| \tau))\right\}
\end{gathered}
$$

where $L=L\left[X^{\mu}, \dot{X}^{\mu}\right]$ is the Lagrangian

$$
L=L\left[X^{\mu}, \dot{X}^{\mu}\right]=-\frac{T}{2} \int_{0}^{\pi} d \sigma\left(-\dot{X}^{\mu} \dot{X}_{\mu}+X^{\prime \mu} X_{\mu}^{\prime}\right) .
$$

Then the standard Poisson brackets

$$
\begin{gathered}
\left\{X^{\mu}(\tau, \sigma), \Pi^{\nu}\left(\tau, \sigma^{\prime}\right)\right\}=\eta^{\mu \nu} \delta\left(\sigma-\sigma^{\prime}\right), \\
\left\{X^{\mu}(\tau, \sigma), X^{\nu}\left(\tau, \sigma^{\prime}\right)\right\}=0=\left\{\Pi^{\mu}(\tau, \sigma), \Pi^{\nu}\left(\tau, \sigma^{\prime}\right)\right\}
\end{gathered}
$$

lead to the following Poisson brackets for $x^{\mu}, p^{\mu}, a_{n}^{\mu}$ and $a_{n}^{\mu *}$

$$
\left\{x^{\mu}, p^{\nu}\right\}=\eta^{\mu \nu}, \quad\left\{a_{m}^{\mu}, a_{n}^{\nu *}\right\}=-\frac{i}{\hbar} \delta_{m n} \eta^{\mu \nu}
$$


with the remaining independent Poisson brackets being zero.

From Eqs. (2.12) and (2.13) we get

$$
x^{\mu}=\frac{1}{\pi} \int_{0}^{\pi} d \sigma X^{\mu}(0, \sigma) \quad \text { and } \quad p^{\mu}=\int_{0}^{\pi} d \sigma \Pi^{\mu}(\tau, \sigma) .
$$

Thus $x^{\mu}$ and $p^{\mu}$ are the the center-of-mass position at $\tau=0$ and momentum of the string. Consequently

$$
M^{2}=-p^{\mu} p_{\mu}
$$

is the square mass of the string.

In string theory it is convenient to use instead of $a_{n}^{\mu}$ and $a_{n}^{\mu *}$ the following objects

$$
\begin{gathered}
\alpha_{n}^{\mu}=-i \sqrt{\hbar n} a_{n}^{\mu}, \quad \widetilde{\alpha}_{n}^{\mu}=-i \sqrt{\hbar n} a_{-n}^{\mu}, \\
\alpha_{-n}^{\mu}=\alpha_{n}^{\mu *}=i \sqrt{\hbar n} a_{n}^{\mu *}, \quad \widetilde{\alpha}_{-n}^{\mu}=\widetilde{\alpha}_{n}^{\mu *}=i \sqrt{\hbar n} a_{-n}^{\mu *}
\end{gathered}
$$

for $n>0$. Substituting Eq. (2.19) into Eqs. (2.12), (2.13) and (2.16) one has

$$
\begin{gathered}
X^{\mu}(\tau, \sigma)=x^{\mu}+\frac{1}{\pi T} p^{\mu} \tau+\frac{i}{2 \sqrt{\pi T}} \sum_{n \neq 0} \frac{1}{n}\left\{\alpha_{n}^{\mu} \exp (-2 i n(\tau-\sigma))+\widetilde{\alpha}_{n}^{\mu} \exp (-2 i n(\tau+\sigma))\right\}, \\
\Pi^{\mu}(\tau, \sigma)=\frac{1}{\pi} p^{\mu}+\sqrt{\frac{T}{\pi}} \sum_{n \neq 0}\left\{\alpha_{n}^{\mu} \exp (-20)\right.
\end{gathered}
$$

and

$$
\begin{gathered}
\left\{x^{\mu}, p^{\nu}\right\}=\eta^{\mu \nu} \\
\left\{\alpha_{m}^{\mu}, \alpha_{n}^{\nu}\right\}=-i m \delta_{m+n, 0} \eta^{\mu \nu}, \quad\left\{\widetilde{\alpha}_{m}^{\mu}, \widetilde{\alpha}_{n}^{\nu}\right\}=-i m \delta_{m+n, 0} \eta^{\mu \nu}
\end{gathered}
$$

for all $m, n \neq 0$. 
We now consider the so called light-cone gauge in which the constraint equations (2.8) can be easily solved and then eliminated. This gauge will be crucial for the deformation quantization of the bosonic string.

First, introduce the light-cone (null) coordinates

$$
X^{ \pm}:=\frac{1}{\sqrt{2}}\left(X^{0} \pm X^{D-1}\right)
$$

and the remaining coordinates $X^{j}, j=1, \ldots, D-2$ are left as before. As $X^{+}(\tau, \sigma)$ satisfies the wave equation (2.7) one can choose the coordinate $\tau$ in such a manner that

$$
X^{+}(\tau, \sigma)=\frac{1}{\pi T} p^{+} \tau
$$

and (2.6) still holds true. In this gauge the constraint equations (2.8) are equivalent to the following equations

$$
\begin{aligned}
& \left(\dot{X}^{\mu}-X^{\prime \mu}\right)\left(\dot{X}_{\mu}-X_{\mu}^{\prime}\right)=0 \Longleftrightarrow \sum_{j=1}^{D-2}\left(\dot{X}^{j}-X^{\prime j}\right)^{2}-\frac{2 p^{+}}{\pi T}\left(\dot{X}^{-}-X^{\prime-}\right)=0, \\
& \left(\dot{X}^{\mu}+X^{\prime \mu}\right)\left(\dot{X}_{\mu}+X_{\mu}^{\prime}\right)=0 \Longleftrightarrow \sum_{j=1}^{D-2}\left(\dot{X}^{j}+X^{\prime j}\right)^{2}-\frac{2 p^{+}}{\pi T}\left(\dot{X}^{-}+X^{\prime-}\right)=0 .
\end{aligned}
$$

Expressing $X^{-}(\tau, \sigma)$ in the form

$X^{-}(\tau, \sigma)=x^{-}+\frac{1}{\pi T} p^{-} \tau+\frac{i}{2 \sqrt{\pi T}} \sum_{n \neq 0} \frac{1}{n}\left\{\alpha_{n}^{-} \exp (-2 i n(\tau-\sigma))+\widetilde{\alpha}_{n}^{-} \exp (-2 i n(\tau+\sigma))\right\}$

and inserting (2.27) into (2.25) and (2.26) one can solve these constraint equations in the sense that $p^{-}, \alpha_{n}^{-}$and $\widetilde{\alpha}_{n}^{-}$are defined by $p^{+}, p^{j}, \alpha_{n}^{j}$ and $\widetilde{\alpha}_{n}^{j}$. Simple calculations give

$$
\alpha_{m}^{-}=\frac{\sqrt{\pi T}}{p^{+}} \sum_{j=1}^{D-2} \sum_{n=-\infty}^{\infty} \alpha_{n}^{j} \alpha_{m-n}^{j}
$$

and 


$$
\widetilde{\alpha}_{m}^{-}=\frac{\sqrt{\pi T}}{p^{+}} \sum_{j=1}^{D-2} \sum_{n=-\infty}^{\infty} \widetilde{\alpha}_{n}^{j} \widetilde{\alpha}_{m-n}^{j}
$$

for all $m \in \mathbb{Z}$ and where we denote

$$
\alpha_{0}^{-}=\widetilde{\alpha}_{0}^{-}:=\frac{1}{2 \sqrt{\pi T}} p^{-} \quad \text { and } \quad \alpha_{0}^{j}=\widetilde{\alpha}_{0}^{j}:=\frac{1}{2 \sqrt{\pi T}} p^{j} .
$$

Thus the (independent) dynamical variables of the string are: $x^{-}, p^{+}, x^{j}, p^{j}, \alpha_{n}^{j}$ and $\widetilde{\alpha}_{n}^{j}$ for $n \neq 0$ or, equivalently: $x^{-}, p^{+}, X^{j}$ and $\Pi^{j}$.

For the Poisson bracket for $x^{-}$and $p^{+}$we have

$$
\left\{x^{-}, p^{+}\right\}=-1
$$

(Note the opposite sign in (2.31) in comparison with the usual one, $\left\{x^{j}, p^{k}\right\}=\delta^{j k}$ ).

The square mass $M^{2}$ given by (2.18) takes now the form

$$
\begin{gathered}
M^{2}=2 p^{+} p^{-}-\sum_{j=1}^{D-2} p^{j} p^{j}=4 \pi T \sum_{j=1}^{D-2} \sum_{n=-\infty}^{\infty} \alpha_{n}^{j} \alpha_{-n}^{j}-4 \pi T \sum_{j=1}^{D-2} \alpha_{0}^{j} \alpha_{0}^{j} \\
=4 \pi T \sum_{j=1}^{D-2} \sum_{n \neq 0} \alpha_{n}^{j} \alpha_{-n}^{j}=4 \pi T \sum_{j=1}^{D-2} \sum_{n \neq 0} \widetilde{\alpha}_{n}^{j} \widetilde{\alpha}_{-n}^{j} .
\end{gathered}
$$

Then the Hamiltonian

$$
H=\frac{T}{2} \int_{0}^{\pi} d \sigma\left\{\sum_{j=1}^{D-2}\left(\left(\frac{\Pi^{j}}{T}\right)^{2}+\left(X^{\prime j}\right)^{2}\right)\right\}
$$

is

$$
\begin{gathered}
H=\sum_{j=1}^{D-2} \sum_{n=-\infty}^{\infty}\left(\alpha_{n}^{j} \alpha_{-n}^{j}+\widetilde{\alpha}_{n}^{j} \widetilde{\alpha}_{-n}^{j}\right) \\
=2 \sum_{j=1}^{D-2} \sum_{n=-\infty}^{\infty} \alpha_{n}^{j} \alpha_{-n}^{j} \\
=\frac{\sum_{j=1}^{D-2}\left(p^{j}\right)^{2}}{2 \pi T}+4 \sum_{j=1}^{D-2} \sum_{n=1}^{\infty} \alpha_{n}^{j} \alpha_{-n}^{j}
\end{gathered}
$$




$$
=\frac{\sum_{j=1}^{D-2}\left(p^{j}\right)^{2}}{2 \pi T}+2 \hbar \sum_{j=1}^{D-2} \sum_{n \neq 0}|n| a_{n}^{j *} a_{n}^{j}=\frac{p^{+} p^{-}}{\pi T} .
$$

From Eqs. (2.28), (2.29) and (2.22) one gets

$$
\begin{aligned}
& \left\{\alpha_{m}^{-}, \alpha_{n}^{-}\right\}=-i \frac{2 \sqrt{\pi T}}{p^{+}}(m-n) \alpha_{m+n}^{-}, \\
& \left\{\widetilde{\alpha}_{m}^{-}, \widetilde{\alpha}_{n}^{-}\right\}=-i \frac{2 \sqrt{\pi T}}{p^{+}}(m-n) \widetilde{\alpha}_{m+n}^{-}
\end{aligned}
$$

for all $m, n \neq 0$. Therefore $\left\{\alpha_{m}^{-}\right.$; for all $\left.m \neq 0\right\}$ and $\left\{\widetilde{\alpha}_{m}^{-}\right.$; for all $\left.m \neq 0\right\}$ constitute the Virasoro algebra without a central extension. Now analogously as in the case of classical fields $[19,18]$ we introduce the oscillator variables $Q_{n}^{j}$ and $P_{n}^{j}, n \neq 0$, as follows

$$
\begin{aligned}
& Q_{n}^{j}(\tau):=\sqrt{\frac{\hbar}{4|n|}}\left(a_{n}^{j}(\tau)+a_{n}^{j *}(\tau)\right) \\
& P_{n}^{j}(\tau):=i \sqrt{\hbar|n|}\left(a_{n}^{j *}(\tau)-a_{n}^{j}(\tau)\right),
\end{aligned}
$$

where $a_{n}^{j}(\tau):=a_{n}^{j} \exp (-2 i|n| \tau)$.

By Eq. 2.16) one has

$$
\begin{gathered}
\left\{Q_{m}^{j}(\tau), P_{n}^{k}(\tau)\right\}=\delta^{j k} \delta_{m n}, \\
\left\{Q_{m}^{j}(\tau), Q_{n}^{k}(\tau)\right\}=0=\left\{P_{m}^{j}(\tau), P_{n}^{k}(\tau)\right\} .
\end{gathered}
$$

From Eq. (2.36) we quickly find

$$
a_{n}^{j}(\tau)=\sqrt{\frac{|n|}{\hbar}}\left(Q_{n}^{j}(\tau)+\frac{i}{2|n|} P_{n}^{j}(\tau)\right) .
$$

Straightforward calculations show that Eqs. (2.12) and (2.13) give

$$
a_{n}^{j}(\tau)=\frac{1}{2 \sqrt{\pi \hbar|n|}} \int_{0}^{\pi} d \sigma\left(2|n| \sqrt{T} X^{j}(\tau, \sigma)+\frac{i}{\sqrt{T}} \Pi^{j}(\tau, \sigma)\right) \exp (-2 i n \sigma)
$$


Substituting Eq. (2.39) into Eq. (2.36) one gets

$$
\begin{gathered}
Q_{n}^{j}(\tau)=\frac{1}{\sqrt{\pi}} \int_{0}^{\pi} d \sigma\left(\sqrt{T} X^{j}(\sigma) \cos (2 n \sigma+2|n| \tau)+\frac{1}{2|n| \sqrt{T}} \Pi^{j}(\sigma) \sin (2 n \sigma+2|n| \tau)\right), \\
P_{n}^{j}(\tau)=\frac{1}{\sqrt{\pi}} \int_{0}^{\pi} d \sigma\left(-2|n| \sqrt{T} X^{j}(\sigma) \sin (2 n \sigma+2|n| \tau)+\frac{1}{\sqrt{T}} \Pi^{j}(\sigma) \cos (2 n \sigma+2|n| \tau)\right),
\end{gathered}
$$

where $X^{j}(\sigma) \equiv X^{j}(0, \sigma)$ and $\Pi^{j}(\sigma) \equiv \Pi^{j}(0, \sigma)$.

Inserting Eq. (2.38) into (2.12) and (2.13) we obtain

$$
\begin{gathered}
X^{j}(\tau, \sigma)=x^{j}+\frac{1}{\pi T} p^{j} \tau+\frac{1}{\sqrt{\pi T}} \sum_{n \neq 0}\left(Q_{n}^{j} \cos (2 n \sigma-2|n| \tau)-\frac{1}{2|n|} P_{n}^{j} \sin (2 n \sigma-2|n| \tau)\right), \\
\Pi^{j}(\tau, \sigma)=\frac{1}{\pi} p^{j}+\sqrt{\frac{T}{\pi}} \sum_{n \neq 0}\left(2|n| Q_{n}^{j} \sin (2 n \sigma-2|n| \tau)+P_{n}^{j} \cos (2 n \sigma-2|n| \tau)\right),
\end{gathered}
$$

where $Q_{n}^{j} \equiv Q_{n}^{j}(0)$ and $P_{n}^{j} \equiv P_{n}^{j}(0)$.

Observe also that from Eq. (2.36) one quickly finds that

$$
\begin{gathered}
Q_{n}^{j}(\tau)=Q_{n}^{j} \cos (2|n| \tau)+\frac{1}{2|n|} P_{n}^{j} \sin (2|n| \tau), \\
P_{n}^{j}(\tau)=-2|n| Q_{n}^{j} \sin (2|n| \tau)+P_{n}^{j} \cos (2|n| \tau) .
\end{gathered}
$$

Finally, Eqs. (2.32) and (2.34) with (2.38) give

$$
M^{2}=4 \pi T \sum_{j=1}^{D-2} \sum_{n \neq 0}\left(\left(P_{n}^{j}\right)^{2}+4 n^{2}\left(Q_{n}^{j}\right)^{2}\right)
$$

and

$$
H=\frac{\sum_{j=1}^{D-2}\left(p^{j}\right)^{2}}{2 \pi T}+\frac{1}{2} \sum_{j=1}^{D-2} \sum_{n \neq 0}\left(\left(P_{n}^{j}\right)^{2}+4 n^{2}\left(Q_{n}^{j}\right)^{2}\right) .
$$


We arrive at the conclusion that one can use the (independent) dynamical variables $\left(x^{-}, p^{+}, x^{j}, p^{j}, Q_{n}^{j}, P_{n}^{j}\right)$ and these variables are canonically related to the variables $\left(x^{-}, p^{+}, X^{j}, \Pi^{j}\right)$.

[Straightforward calculations give

$$
\begin{gathered}
\left\{X^{j}(\tau, \sigma), \Pi^{k}\left(\tau, \sigma^{\prime}\right)\right\}_{(x, p, Q, P)}:=\sum_{l=1}^{D-2}\left\{\left(\frac{\partial X^{j}(\tau, \sigma)}{\partial x^{l}} \frac{\partial \Pi^{k}\left(\tau, \sigma^{\prime}\right)}{\partial p^{l}}-\frac{\partial X^{j}(\tau, \sigma)}{\partial p^{l}} \frac{\partial \Pi^{k}\left(\tau, \sigma^{\prime}\right)}{\partial x^{l}}\right)+\right. \\
\left.\sum_{n \neq 0}\left(\frac{\partial X^{j}(\tau, \sigma)}{\partial Q_{n}^{l}} \frac{\partial \Pi^{k}\left(\tau, \sigma^{\prime}\right)}{\partial P_{n}^{l}}-\frac{\partial X^{j}(\tau, \sigma)}{\partial P_{n}^{l}} \frac{\partial \Pi^{k}\left(\tau, \sigma^{\prime}\right)}{\partial Q_{n}^{l}}\right)\right\} \\
=\frac{1}{\pi} \delta^{j k}+\delta^{j k}\left(\delta\left(\sigma-\sigma^{\prime}\right)-\frac{1}{\pi}\right)=\delta^{j k} \delta\left(\sigma-\sigma^{\prime}\right), \\
\left.\left\{X^{j}(\tau, \sigma), X^{k}\left(\tau, \sigma^{\prime}\right)\right\}_{(x, p, Q, P)}=0=\left\{\Pi^{j}(\tau, \sigma), \Pi^{k}\left(\tau, \sigma^{\prime}\right)\right\}_{(x, p, Q, P)} .\right]
\end{gathered}
$$

\subsection{Open Strings}

In this case the general solution of Eqs. (2.7) satisfying the boundary conditions (2.10) can be represented by the series

$$
\begin{aligned}
X^{\mu}(\tau, \sigma)=x^{\mu} & +\frac{1}{\pi T} p^{\mu} \tau+\frac{1}{\sqrt{\pi T}} \sum_{n=1}^{\infty} \sqrt{\frac{\hbar}{n}}\left(a_{n}^{\mu} \exp (-i n \tau)+a_{n}^{\mu *} \exp (i n \tau)\right) \cos (n \sigma) \\
& =x^{\mu}+\frac{1}{\pi T} p^{\mu} \tau+\frac{i}{\sqrt{\pi T}} \sum_{n \neq 0} \frac{1}{n} \alpha_{n}^{\mu} \exp (-i n \tau) \cos (n \sigma)
\end{aligned}
$$

Note that the boundary condition (2.10) at $\sigma=0$ yields $a_{n}^{\mu}=a_{-n}^{\mu}$ for all $n \neq 0$. Here $\alpha_{n}^{\mu}$ are defined as before by $(2.19)$ and $\widetilde{\alpha}_{n}^{\mu}$ do not appear as independent variables because $a_{n}^{\mu}=a_{-n}^{\mu}$.

Then

$$
\Pi^{\mu}(\tau, \sigma)=T \dot{X}^{\mu}=\frac{1}{\pi} p^{\mu}+i \sqrt{\frac{T}{\pi}} \sum_{n=1}^{\infty} \sqrt{\hbar n}\left(a_{n}^{\mu *} \exp (i n \tau)-a_{n}^{\mu} \exp (-i n \tau)\right) \cos (n \sigma)
$$




$$
=\frac{1}{\pi} p^{\mu}+\sqrt{\frac{T}{\pi}} \sum_{n \neq 0} \alpha_{n}^{\mu} \exp (-i n \tau) \cos (n \sigma) .
$$

In the light-cone gauge we have

$$
\begin{gathered}
X^{+}=\frac{1}{\pi T} p^{+} \tau \\
X^{-}=x^{-}+\frac{1}{\pi T} p^{-} \tau+\frac{i}{\sqrt{\pi T}} \sum_{n \neq 0} \frac{1}{n} \alpha_{n}^{\mu} \exp (-i n \tau) \cos (n \sigma) .
\end{gathered}
$$

Then the solution of the constraint equations (2.8) or, equivalently, (2.25) and (2.26) reads

$$
\alpha_{m}^{-}=\frac{\sqrt{\pi T}}{2 p^{+}} \sum_{j=1}^{D-2} \sum_{n=-\infty}^{\infty} \alpha_{n}^{j} \alpha_{m-n}^{j}
$$

for all $m \in \mathbb{Z}$, where now

$$
\alpha_{0}^{-}:=\frac{1}{\sqrt{\pi T}} p^{-} \quad \text { and } \quad \alpha_{0}^{j}:=\frac{1}{\sqrt{\pi T}} p^{j} .
$$

(Compare with Eq. (2.30).)

The square mass $M^{2}$ is

$$
M^{2}=-p^{\mu} p_{\mu}=2 p^{+} p_{-}-\sum_{j=1}^{D-2} p^{j} p^{j}=\pi T \sum_{j=1}^{D-2} \sum_{n \neq 0} \alpha_{n}^{j} \alpha_{-n}^{j}
$$

and the Hamiltonian (2.33) reads

$$
\begin{gathered}
H=\frac{1}{2} \sum_{j=1}^{D-2} \sum_{n=-\infty}^{\infty} \alpha_{n}^{j} \alpha_{-n}^{j} \\
=\frac{\sum_{j=1}^{D-2}\left(p^{j}\right)^{2}}{2 \pi T}+\hbar \sum_{j=1}^{D-2} \sum_{n=1}^{\infty} n a_{n}^{j} a_{n}^{j *} .
\end{gathered}
$$

For the Poisson brackets $\left\{\alpha_{m}^{-}, \alpha_{n}^{-}\right\}$one gets

$$
\left\{\alpha_{m}^{-}, \alpha_{n}^{-}\right\}=-i \frac{\sqrt{\pi T}}{p^{+}}(m-n) \alpha_{m+n}^{-}
$$


for all $m, n \neq 0$. (Compare with (2.35)).

Analogously as before we introduce new variables $Q_{n}^{j}$ and $P_{n}^{j}, n=1, \ldots, \infty$

$$
\begin{aligned}
& Q_{n}^{j}(\tau):=\sqrt{\frac{\hbar}{2 n}}\left(a_{n}^{j}(\tau)+a_{n}^{j *}(\tau)\right)=\frac{i}{n \sqrt{2}}\left(\alpha_{n}^{j}(\tau)-\alpha_{-n}^{j}(\tau)\right) \\
& P_{n}^{j}(\tau):=i \sqrt{\frac{\hbar n}{2}}\left(a_{n}^{j *}(\tau)-a_{n}^{j}(\tau)\right)=\frac{1}{\sqrt{2}}\left(\alpha_{n}^{j}(\tau)+\alpha_{-n}^{j}(\tau)\right),
\end{aligned}
$$

where $a_{n}^{j}(\tau):=a_{n}^{j} \exp (-i n \tau), n \in \mathbb{Z}_{+} \cdot Q_{n}^{j}(\tau)$ and $P_{n}^{j}(\tau)$ fulfill the Poisson bracket formulas (2.37). Then

$$
a_{n}^{j}(\tau)=\sqrt{\frac{n}{2 \hbar}}\left(Q_{n}^{j}(\tau)+\frac{i}{n} P_{n}^{j}(\tau)\right), \quad n \in \mathbb{Z}_{+} .
$$

Inserting Eq. (2.55) into Eqs. (2.46) and (2.47) one gets

$$
\begin{aligned}
& X^{j}(\tau, \sigma)=x^{j}+\frac{1}{\pi T} p^{j} \tau+\sqrt{\frac{2}{\pi T}} \sum_{n=1}^{\infty}\left(Q_{n}^{j} \cos (n \tau)+\frac{1}{n} P_{n}^{j} \sin (n \tau)\right) \cos (n \sigma), \\
& \Pi^{j}(\tau, \sigma)=\frac{1}{\pi} p^{j}+\sqrt{\frac{2 T}{\pi}} \sum_{n=1}^{\infty}\left(-n Q_{n}^{j} \sin (n \tau)+P_{n}^{j} \cos (n \tau)\right) \cos (n \sigma),
\end{aligned}
$$

where, as before, $Q_{n}^{j} \equiv Q_{n}^{j}(0)$ and $P_{n}^{j} \equiv P_{n}^{j}(0)$.

From Eqs. (2.46) and (2.47) we find

$$
a_{n}^{j}(\tau)=\frac{1}{\sqrt{\pi \hbar n}} \int_{0}^{\pi} d \sigma\left(n \sqrt{T} X^{j}(\tau, \sigma)+\frac{i}{\sqrt{T}} \Pi^{j}(\tau, \sigma)\right) \cos (n \sigma) .
$$

Substituting Eq. (2.57) into (2.54) one quickly obtains

$$
\begin{gathered}
Q_{n}^{j}(\tau)=\sqrt{\frac{2}{\pi}} \int_{0}^{\pi} d \sigma\left(\sqrt{T} X^{j}(\sigma) \cos (n \tau)+\frac{1}{n \sqrt{T}} \Pi^{j}(\sigma) \sin (n \tau)\right) \cos (n \sigma), \\
P_{n}^{j}(\tau)=\sqrt{\frac{2}{\pi}} \int_{0}^{\pi} d \sigma\left(-n \sqrt{T} X^{j}(\sigma) \sin (n \tau)+\frac{1}{\sqrt{T}} \Pi^{j}(\sigma) \cos (n \tau)\right) \cos (n \sigma) .
\end{gathered}
$$


From Eq. (2.58) we have

$$
\begin{aligned}
& Q_{n}^{j}(\tau)=Q_{n}^{j} \cos (n \tau)+\frac{1}{n} P_{n}^{j} \sin (n \tau), \\
& P_{n}^{j}(\tau)=-n Q_{n}^{j} \sin (n \tau)+P_{n}^{j} \cos (n \tau),
\end{aligned}
$$

(compare with Eq. (2.42)).

Finally, for $M^{2}$ and $H$ one gets

$$
M^{2}=\pi T \sum_{j=1}^{D-2} \sum_{n=1}^{\infty}\left(\left(P_{n}^{j}\right)^{2}+n^{2}\left(Q_{n}^{j}\right)^{2}\right)
$$

and

$$
H=\frac{\sum_{j=1}^{D-2}\left(p^{j}\right)^{2}}{2 \pi T}+\frac{1}{2} \sum_{j=1}^{D-2} \sum_{n=1}^{\infty}\left(\left(P_{n}^{j}\right)^{2}+n^{2}\left(Q_{n}^{j}\right)^{2}\right) .
$$

As before we can use the (independent) dynamical variables $\left(x^{-}, p^{+}, x^{j}, p^{j}, Q_{n}^{j}, P_{n}^{j}\right)$ and they are canonically related to $\left(x^{-}, p^{+}, X^{j}, \Pi^{j}\right)$. Observe that in the present case $n \in \mathbb{Z}_{+}$.

\section{Deformation Quantization of the Bosonic String}

In this section we are going to use the well known machinery of deformation quantization [20-31] to the case of bosonic strings.

\subsection{Closed Strings}

According to results of subsection 2.1 the phase space $\mathcal{Z}$ of a closed string can be

understood as the Cartesian product $\mathcal{Z}=\mathbb{R}^{2} \times \mathbb{R}^{2(D-2)} \times \mathbb{R}^{2 \infty}$ endowed with the following symplectic form

$$
\omega=d p_{-} \wedge d x^{-}+\sum_{j=1}^{D-2}\left(d p_{j} \wedge d x^{j}+\sum_{n \neq 0} d P_{j n} \wedge d Q_{n}^{j}\right)
$$


where $p_{-}=-p^{+}, p_{j}=p^{j}$ and $P_{j n}=P_{n}^{j}$.

Equivalently, one can consider $\mathcal{Z}$ to be $\mathcal{Z}=\mathbb{R}^{2} \times \Gamma$ where $\Gamma$ is the set $\Gamma=$ $\left\{\left(X^{j}(\sigma), \Pi_{j}(\sigma)\right)_{j=1, \ldots, D-2}\right\}$ with $X^{j}(\sigma)$ and $\Pi^{j}(\sigma)=\Pi_{j}(\sigma)$ being arbitrary real functions of $\sigma \in[0, \pi]$ satisfying the boundary conditions: $X^{j}(0)=X^{j}(\pi)$ and $\Pi^{j}(0)=\Pi^{j}(\pi)$. The symplectic form has now the functional form

$$
\omega=d p_{-} \wedge d x^{-}+\sum_{j=1}^{D-2} \int_{0}^{\pi} d \sigma \delta \Pi_{j}(\sigma) \wedge \delta X^{j}(\sigma)
$$

Let $\hat{x}^{-}, \hat{p}^{+}=-\hat{p}_{-}, \hat{X}^{j}$ and $\hat{\Pi}^{j}$ be the field operators

$$
\begin{gathered}
\hat{x}^{-}\left|x^{-}\right\rangle=x^{-}\left|x^{-}\right\rangle, \quad \hat{p}^{+}\left|p^{+}\right\rangle=p^{+}\left|p^{+}\right\rangle, \\
\hat{X}^{j}(\sigma)\left|X^{j}\right\rangle=X^{j}(\sigma)\left|X^{j}\right\rangle, \quad \hat{\Pi}^{j}(\sigma)\left|\Pi^{j}\right\rangle=\Pi^{j}(\sigma)\left|\Pi^{j}\right\rangle, \\
{\left[\hat{X}^{j}(\sigma), \hat{\Pi}^{k}\left(\sigma^{\prime}\right)\right]=i \hbar \delta^{j k} \delta\left(\sigma-\sigma^{\prime}\right), \quad\left[\hat{x}^{-}, \hat{p}^{+}\right]=-i \hbar .}
\end{gathered}
$$

Denote

$$
\begin{array}{r}
\left|x^{-}, X\right\rangle:=\left|x^{-}\right\rangle \otimes\left(\bigotimes_{j=1}^{D-2}\left|X^{j}\right\rangle\right), \quad\left|p^{+}, \Pi\right\rangle:=\left|p^{+}\right\rangle \otimes\left(\bigotimes_{j=1}^{D-2}\left|\Pi^{j}\right\rangle\right), \\
\mathcal{D} X=\prod_{\sigma} d X^{1}(\sigma) \ldots d X^{D-2}(\sigma) \quad \text { and } \quad \mathcal{D} \Pi=\prod_{\sigma} d \Pi^{1}(\sigma) \ldots d \Pi^{D-2}(\sigma) .
\end{array}
$$

Then we fix the normalization as follows

$$
\int d x^{-} \mathcal{D} X\left|x^{-}, X\right\rangle\left\langle x^{-}, X\right|=\hat{1} \quad \text { and } \quad \int d\left(\frac{p^{+}}{2 \pi \hbar}\right) \mathcal{D}\left(\frac{\Pi}{2 \pi \hbar}\right)\left|p^{+}, \Pi\right\rangle\left\langle p^{+}, \Pi\right|=\hat{1} .
$$

Let $F=F\left[x^{-}, X, p^{+}, \Pi\right]$ be a functional on the phase space $\mathcal{Z}$. Then according to the Weyl rule we assign the following operator $\hat{F}$ corresponding to $F$ 


$$
\hat{F}=W(F)=\int \frac{d x^{-} d p^{+}}{2 \pi \hbar} \mathcal{D} X \mathcal{D}\left(\frac{\Pi}{2 \pi \hbar}\right) F\left[x^{-}, X, p^{+}, \Pi\right] \hat{\Omega}\left[x^{-}, X, p^{+}, \Pi\right]
$$

where $\hat{\Omega}\left[x^{-}, X, p^{+}, \Pi\right]$ is the Stratonovich-Weyl quantizer (SW)

$$
\begin{gathered}
\hat{\Omega}\left[x^{-}, X, p^{+}, \Pi\right]=\int d \xi^{-} \mathcal{D} \xi \exp \left\{-\frac{i}{\hbar}\left(-\xi^{-} p^{+}+\int_{0}^{\pi} d \sigma \xi(\sigma) \cdot \Pi(\sigma)\right)\right\} \\
\left|x^{-}-\frac{\xi^{-}}{2}, X-\frac{\xi}{2}\right\rangle\left\langle X+\frac{\xi}{2}, x^{-}+\frac{\xi^{-}}{2}\right| \\
=\int d\left(\frac{\eta^{+}}{2 \pi \hbar}\right) \mathcal{D}\left(\frac{\eta}{2 \pi \hbar}\right) \exp \left\{-\frac{i}{\hbar}\left(-x^{-} \eta^{+}+\int_{0}^{\pi} d \sigma \eta(\sigma) \cdot X(\sigma)\right)\right\}\left|p^{+}+\frac{\eta^{+}}{2}, \Pi+\frac{\eta}{2}\right\rangle\left\langle\Pi-\frac{\eta}{2}, p^{+}-\frac{\eta^{+}}{2}\right|
\end{gathered}
$$

with the obvious notation $\xi(\sigma) \cdot \Pi(\sigma) \equiv \sum_{j=1}^{D-2} \xi^{j}(\sigma) \Pi^{j}(\sigma)$ and $\eta(\sigma) \cdot X(\sigma) \equiv \sum_{j=1}^{D-2} \eta^{j}(\sigma) X^{j}(\sigma)$.

The SW quantizer has the properties

$$
\begin{gathered}
\left(\hat{\Omega}\left[x^{-}, X, p^{+}, \Pi\right]\right)^{\dagger}=\hat{\Omega}\left[x^{-}, X, p^{+}, \Pi\right] \\
\operatorname{Tr}\left(\hat{\Omega}\left[x^{-}, X, p^{+}, \Pi\right]\right)=1 \\
\operatorname{Tr}\left(\hat{\Omega}\left[x^{-}, X, p^{+}, \Pi\right] \hat{\Omega}\left[{ }^{\prime} x^{-},{ }^{\prime} X,{ }^{\prime} p^{+},{ }^{\prime} \Pi\right]\right)=\delta\left(x^{-}-{ }^{\prime} x^{-}\right) \delta\left(\frac{p^{+}-{ }^{\prime} p^{+}}{2 \pi \hbar}\right) \delta\left[X-{ }^{\prime} X\right] \delta\left[\frac{\Pi-{ }^{\prime} \Pi}{2 \pi \hbar}\right]
\end{gathered}
$$

Multiplying Eq. (3.6) by $\hat{\Omega}\left[x^{-}, X, p^{+}, \Pi\right]$ and taking the trace one has

$$
W^{-1}(\hat{F})=F\left[x^{-}, X, p^{+}, \Pi\right]=\operatorname{Tr}\left(\hat{\Omega}\left[x^{-}, X, p^{+}, \Pi\right] \hat{F}\right) .
$$

This enables us to solve the following problem. Let $F_{1}=F_{1}\left[x^{-}, X, p^{+}, \Pi\right]$ and $F_{2}=$ $F_{2}\left[x^{-}, X, p^{+}, \Pi\right]$ be functionals defined on the phase space $\mathcal{Z}$ and let $\hat{F}_{1}=W\left(F_{1}\right)$ and $\hat{F}_{2}=W\left(F_{2}\right)$ be their corresponding operators. The problem is what a functional on $\mathcal{Z}$ 
corresponds to the product $\hat{F}_{1} \hat{F}_{2}$. This functional is denoted by $F_{1} * F_{2}$ and it is called the Moyal $*$-product of $F_{1}$ and $F_{2}$.

By Eq. (3.11) one gets

$$
\left(F_{1} * F_{2}\right)\left[x^{-}, X, p^{+}, \Pi\right]:=W^{-1}\left(\hat{F}_{1} \hat{F}_{2}\right)=\operatorname{Tr}\left(\hat{\Omega}\left[x^{-}, X, p^{+}, \Pi\right] \hat{F}_{1} \hat{F}_{2}\right) .
$$

Substituting Eq. (3.6) into (3.12), using then (3.7) and perfoming straightforward but tedious manipulations (see for example [30]) we finally obtain

$$
\begin{gathered}
\left(F_{1} * F_{2}\right)\left[x^{-}, X, p^{+}, \Pi\right]=F_{1}\left[x^{-}, X, p^{+}, \Pi\right] \exp \left\{\frac{i \hbar}{2} \overleftrightarrow{\mathcal{P}}\right\} F_{2}\left[x^{-}, X, p^{+}, \Pi\right] \\
\overleftrightarrow{\mathcal{P}}:=\left(\frac{\overleftarrow{\partial}}{\partial p^{+}} \frac{\vec{\partial}}{\partial x^{-}}-\frac{\overleftarrow{\partial}}{\partial x^{-}} \frac{\vec{\partial}}{\partial p^{+}}\right)+\sum_{j=1}^{D-2} \int_{0}^{\pi} d \sigma\left(\frac{\overleftarrow{\delta}}{\delta X^{j}(\sigma)} \frac{\vec{\delta}}{\delta \Pi^{j}(\sigma)}-\frac{\overleftrightarrow{\delta}}{\delta \Pi^{j}(\sigma)} \frac{\vec{\delta}}{\delta X^{j}(\sigma)}\right)
\end{gathered}
$$

Now it is an easy matter to define the Wigner functional. Assume $\hat{\rho}$ to be the density operator of the quantum state of a bosonic string. Then according to the general formula (3.11) the functional $\rho\left[x^{-}, X, p^{+}, \Pi\right]$ corresponding to $\hat{\rho}$ reads (use also (3.7))

$$
\begin{gathered}
\rho\left[x^{-}, X, p^{+}, \Pi\right]=W^{-1}(\hat{\rho})=\operatorname{Tr}\left(\hat{\Omega}\left[x^{-}, X, p^{+}, \Pi\right] \hat{\rho}\right) \\
=\int d \xi^{-} \mathcal{D} \xi \exp \left\{-\frac{i}{\hbar}\left(-\xi^{-} p^{+}+\int_{0}^{\pi} d \sigma \xi(\sigma) \cdot \Pi(\sigma)\right)\right\}\left\langle X+\frac{\xi}{2}, x^{-}+\frac{\xi^{-}}{2}|\hat{\rho}| x^{-}-\frac{\xi^{-}}{2}, X-\frac{\xi}{2}\right\rangle .
\end{gathered}
$$

Then the Wigner functional $\rho_{W}\left[x^{-}, X, p^{+}, \Pi\right]$ is defined by a simple modification of Eq. (3.14). Namely,

$$
\begin{gathered}
\rho_{W}\left[x^{-}, X, p^{+}, \Pi\right]:=\int d\left(\frac{\xi^{-}}{2 \pi \hbar}\right) \mathcal{D}\left(\frac{\xi}{2 \pi \hbar}\right) \exp \left\{-\frac{i}{\hbar}\left(-\xi^{-} p^{+}+\int_{0}^{\pi} d \sigma \xi(\sigma) \cdot \Pi(\sigma)\right)\right\} \\
\left\langle X+\frac{\xi}{2}, x^{-}+\frac{\xi^{-}}{2}|\hat{\rho}| x^{-}-\frac{\xi^{-}}{2}, X-\frac{\xi}{2}\right\rangle .
\end{gathered}
$$


In particular for the pure state $\hat{\rho}=|\Psi\rangle\langle\Psi|$ we get

$$
\begin{aligned}
\rho_{W}\left[x^{-}, X, p^{+}, \Pi\right]= & \int d\left(\frac{\xi^{-}}{2 \pi \hbar}\right) \mathcal{D}\left(\frac{\xi}{2 \pi \hbar}\right) \exp \left\{-\frac{i}{\hbar}\left(-\xi^{-} p^{+}+\int_{0}^{\pi} d \sigma \xi(\sigma) \cdot \Pi(\sigma)\right)\right\} \\
& \Psi^{*}\left[x^{-}-\frac{\xi^{-}}{2}, X-\frac{\xi}{2}\right] \Psi\left[x^{-}+\frac{\xi^{-}}{2}, X+\frac{\xi}{2}\right]
\end{aligned}
$$

where $\Psi\left[x^{-}, X\right]$ stands for $|\Psi\rangle$ in the Schödinger representation.

As it will be clear very soon some calculations simplify when the variables $\left(x^{-}, p^{+}, x^{j}, p^{j}\right.$, $\left.Q_{n}^{j}, P_{n}^{j}\right)$ are used. In terms of these variables one has (in the obvious notation)

$$
\begin{aligned}
& \hat{\Omega}\left(x^{-}, Q, p^{+}, P\right)=\int d \xi^{-} d \xi \exp \left\{-\frac{i}{\hbar}\left(-\xi^{-} p^{+}+\xi \cdot P\right)\right\}\left|x^{-}-\frac{\xi^{-}}{2}, Q-\frac{\xi}{2}\right\rangle\left\langle Q+\frac{\xi}{2}, x^{-}+\frac{\xi^{-}}{2}\right| \\
& =\int d\left(\frac{\eta^{+}}{2 \pi \hbar}\right) d\left(\frac{\eta}{2 \pi \hbar}\right) \exp \left\{-\frac{i}{\hbar}\left(-x^{-} \eta^{+}+\eta \cdot Q\right)\right\}\left|p^{+}+\frac{\eta^{+}}{2}, P+\frac{\eta}{2}\right\rangle\left\langle P-\frac{\eta}{2}, p^{+}-\frac{\eta^{+}}{2}\right|,
\end{aligned}
$$

where $d \xi \equiv \prod_{n \in \mathbb{Z}} d \xi_{n}^{1} \ldots d \xi_{n}^{D-2}, d\left(\frac{\eta}{2 \pi \hbar}\right) \equiv \prod_{n \in \mathbb{Z}} d\left(\frac{\eta_{n}^{1}}{2 \pi \hbar}\right) \ldots d\left(\frac{\eta_{n}^{D-2}}{2 \pi \hbar}\right), \xi \cdot P \equiv \sum_{j=1}^{D-2} \sum_{n=-\infty}^{\infty} \xi_{n}^{j} P_{n}^{j}$, $\eta \cdot Q \equiv \sum_{j=1}^{D-2} \sum_{n=-\infty}^{\infty} \eta_{n}^{j} Q_{n}^{j}, P_{0}^{j} \equiv p^{j}$ and $Q_{0}^{j} \equiv x^{j}$.

Then

$$
\begin{gathered}
\left(F_{1} * F_{2}\right)\left(x^{-}, Q, p^{+}, P\right)=F_{1}\left(x^{-}, Q, p^{+}, P\right) \exp \left\{\frac{i \hbar}{2} \overleftrightarrow{\mathcal{P}}\right\} F_{2}\left(x^{-}, Q, p^{+}, P\right) \\
\overleftrightarrow{\mathcal{P}}:=\left(\frac{\overleftarrow{\partial}}{\partial p^{+}} \frac{\vec{\partial}}{\partial x^{-}}-\frac{\overleftarrow{\partial}}{\partial x^{-}} \frac{\vec{\partial}}{\partial p^{+}}\right)+\sum_{j=1}^{D-2} \sum_{n=-\infty}^{\infty}\left(\frac{\overleftarrow{\partial}}{\partial Q_{n}^{j}} \frac{\vec{\partial}}{\partial P_{n}^{j}}-\frac{\overleftarrow{\partial}}{\partial P_{n}^{j}} \frac{\vec{\partial}}{\partial Q_{n}^{j}}\right) .
\end{gathered}
$$

We can also express the Moyal *-product in terms of $a_{n}^{j}$ and $a_{n}^{j *}$ or $\alpha_{n}^{j}$ and $\widetilde{\alpha}_{n}^{j}$ :

$$
\begin{gathered}
*=\exp \left\{\frac{i \hbar}{2} \stackrel{\leftrightarrow}{\mathcal{P}}\right\} \\
=\exp \left\{\frac{i \hbar}{2}\left[\left(\frac{\overleftarrow{\partial}}{\partial p^{+}} \frac{\vec{\partial}}{\partial x^{-}}-\frac{\overleftarrow{\partial}}{\partial x^{-}} \frac{\vec{\partial}}{\partial p^{+}}\right)+\sum_{j=1}^{D-2}\left(\frac{\overleftarrow{\partial}}{\partial x^{j}} \frac{\vec{\partial}}{\partial p^{j}}-\frac{\overleftarrow{\partial}}{\partial p^{j}} \frac{\vec{\partial}}{\partial x^{j}}\right)\right]\right\}
\end{gathered}
$$




$$
\begin{gathered}
\exp \left\{\frac{1}{2} \sum_{j=1}^{D-2} \sum_{n \neq 0}\left(\frac{\overleftarrow{\partial}}{\partial a_{n}^{j}} \frac{\vec{\partial}}{\partial a_{n}^{j *}}-\frac{\overleftarrow{\partial}}{\partial a_{n}^{j *}} \frac{\vec{\partial}}{\partial a_{n}^{j}}\right)\right\} \\
=\ldots \exp \left\{\frac{\hbar}{2} \sum_{j=1}^{D-2} \sum_{n \neq 0} n\left(\frac{\overleftarrow{\partial}}{\partial \alpha_{n}^{j}} \frac{\vec{\partial}}{\partial \alpha_{-n}^{j}}+\frac{\overleftarrow{\partial}}{\partial \widetilde{\alpha}_{n}^{j}} \frac{\vec{\partial}}{\partial \widetilde{\alpha}_{-n}^{j}}\right)\right\} .
\end{gathered}
$$

Finally, for the Wigner function one obtains

$$
\begin{gathered}
\rho_{W}\left(x^{-}, Q, p^{+}, P\right)=\int d\left(\frac{\xi^{-}}{2 \pi \hbar}\right) d\left(\frac{\xi}{2 \pi \hbar}\right) \exp \left\{-\frac{i}{\hbar}\left(-\xi^{-} p^{+}+\xi \cdot P\right)\right\} \\
\left\langle Q+\frac{\xi}{2}, x^{-}+\frac{\xi^{-}}{2}|\hat{\rho}| x^{-}-\frac{\xi^{-}}{2}, Q-\frac{\xi}{2}\right\rangle
\end{gathered}
$$

and in the case of the pure state $\hat{\rho}=|\Psi\rangle\langle\Psi|$

$$
\begin{gathered}
\rho_{W}\left(x^{-}, Q, p^{+}, P\right)=\int d\left(\frac{\xi^{-}}{2 \pi \hbar}\right) d\left(\frac{\xi}{2 \pi \hbar}\right) \exp \left\{-\frac{i}{\hbar}\left(-\xi^{-} p^{+}+\xi \cdot P\right)\right\} \\
\Psi^{*}\left(x^{-}-\frac{\xi^{-}}{2}, Q-\frac{\xi}{2}\right) \Psi\left(x^{-}+\frac{\xi^{-}}{2}, Q+\frac{\xi}{2}\right) .
\end{gathered}
$$

Given $\rho_{W}$ one can use Eq. (3.6) to find the coresponding density operator $\hat{\rho}$

$$
\hat{\rho}=\int d x^{-} d p^{+} d Q d P \rho_{W}\left(x^{-}, Q, p^{+}, P\right) \hat{\Omega}\left(x^{-}, Q, p^{+}, P\right) .
$$

Consequently, the average value $\langle\hat{F}\rangle$ reads

$$
\begin{gathered}
\langle\hat{F}\rangle=\frac{\operatorname{Tr}(\hat{\rho} \hat{F})}{\operatorname{Tr}(\hat{\rho})} \\
=\frac{\int d x^{-} d p^{+} d Q d P \rho_{W}\left(x^{-}, Q, p^{+}, P\right) \operatorname{Tr}\left(\hat{\Omega}\left(x^{-}, Q, p^{+}, P\right) \hat{F}\right)}{\int d x^{-} d p^{+} d Q d P \rho_{W}\left(x^{-}, Q, p^{+}, P\right)} \\
=\frac{\int d x^{-} d p^{+} d Q d P \rho_{W}\left(x^{-}, Q, p^{+}, P\right) W^{-1}(\hat{F})\left(x^{-}, Q, p^{+}, P\right)}{\int d x^{-} d p^{+} d Q d P \rho_{W}\left(x^{-}, Q, p^{+}, P\right)} .
\end{gathered}
$$

Assume that $\hat{\rho}=|\Psi\rangle\langle\Psi|$. Substituting this $\hat{\rho}$ into Eq. (3.22), multiplying from the left by $\left\langle\widetilde{Q}, \widetilde{x}^{-}\right|$and from the right by $\left|\widetilde{x}^{-}, \widetilde{Q}\right\rangle$ and employing Eq. (3.17) one gets 


$$
\left|\Psi\left(\widetilde{x}^{-}, \widetilde{Q}\right)\right|^{2}=\int d p^{+} d P \rho_{W}\left(\widetilde{x}^{-}, \widetilde{Q}, p^{+}, P\right)
$$

Suppose that $\Psi\left(\widetilde{x}^{-}, \widetilde{Q}\right) \neq 0$. Then inserting $\hat{\rho}=|\Psi\rangle\langle\Psi|$ into (3.22), multiplying from the left by $\left\langle Q, x^{-}\right|$and from the right by $\left|\widetilde{x}^{-}, \widetilde{Q}\right\rangle$, using Eqs. (3.17) and (3.24) we easily find the wave function $\Psi\left(x^{-}, Q\right)$ in terms of the corresponding Wigner function $\rho_{W}$

$$
\begin{gathered}
\Psi\left(x^{-}, Q\right)=\exp \{i \varphi\} \\
\frac{\int d p^{+} d P \rho_{W}\left[\frac{x^{-}+\widetilde{x}^{-}}{2}, \frac{Q+\widetilde{Q}}{2}, p^{+}, P\right] \exp \left\{-\frac{i}{\hbar}\left(-\left(x^{-}-\widetilde{x}^{-}\right) p^{+}+(Q-\widetilde{Q}) \cdot P\right)\right\}}{\left(\int d p^{+} d P \rho_{W}\left(\widetilde{x}-, \widetilde{Q}, p^{+}, P\right)\right)^{1 / 2}},
\end{gathered}
$$

where $\varphi$ is an arbitrary real constant.

Of course in terms of $X^{j}(\sigma)$ and $\Pi^{j}(\sigma)$ one has

$$
\begin{gathered}
\Psi\left[x^{-}, X\right]=\exp \{i \varphi\} \\
\frac{\int d p^{+} \mathcal{D} \Pi \rho_{W}\left[\frac{x^{-}+\widetilde{x}^{-}}{2}, \frac{X+\widetilde{X}}{2}, p^{+}, \Pi\right] \exp \left\{-\frac{i}{\hbar}\left(-\left(x^{-}-\widetilde{x}^{-}\right) p^{+}+\int_{0}^{\pi} d \sigma(X(\sigma)-\tilde{X}(\sigma)) \cdot \Pi(\sigma)\right)\right\}}{\left(\int d p^{+} \mathcal{D} \Pi \rho_{W}\left[\widetilde{x}^{-}, \tilde{X}, p^{+}, \Pi\right]\right)^{1 / 2}},
\end{gathered}
$$

where $X(\sigma) \cdot \Pi(\sigma) \equiv \sum_{j=1}^{D-2} X^{j}(\sigma) \Pi^{j}(\sigma)$.

The natural question is: when a real function $\rho_{W}\left(x^{-}, Q, p^{+}, P\right)$ repesents some quantum state, i.e. it can be considered to be a Wigner function. The necessary and sufficient condition reads

$$
\int d x^{-} d p^{+} d Q d P \rho_{W}\left(x^{-}, Q, p^{+}, P\right)\left(f^{*} * f\right)\left(x^{-}, Q, p^{+}, P\right) \geq 0
$$

for any $f \in C^{\infty}(\mathcal{Z})[[\hbar]]$, and

$$
\int d x^{-} d p^{+} d Q d P \rho_{W}\left(x^{-}, Q, p^{+}, P\right)>0
$$

(See [28,31]). 


\subsection{Example: The Ground State}

The Wigner function $\rho_{W 0}$ of the ground state is defined by

$$
a_{n}^{j} * \rho_{W 0}=0, \quad p^{j} * \rho_{W 0}=0 \quad \text { and } \quad p^{+} * \rho_{W 0}=0,
$$

for $j=1, \ldots, D-2$ and $n \neq 0$.

Employing Eq. (3.19) we have

$$
a_{n}^{j} \rho_{W 0}+\frac{1}{2} \frac{\partial \rho_{W 0}}{\partial a_{n}^{j *}}=0, \quad p^{j} \rho_{W 0}=0, \quad \text { and } \quad p^{+} \rho_{W 0}=0,
$$

for $j=1, \ldots, D-2$ and $n \neq 0$. The general real solution of Eq. (3.30) satisfying also Eqs. $(3.27)$ and $(3.28)$ reads

$$
\rho_{W 0}=C \exp \left\{-2 \sum_{j=1}^{D-2} \sum_{n \neq 0} a_{n}^{j} a_{n}^{j *}\right\} \delta\left(p^{1}\right) \ldots \delta\left(p^{D-2}\right) \delta\left(p^{+}\right)
$$

where $C>0$. Consequently, in terms of $Q_{n}^{j}$ and $P_{n}^{j}$ one gets

$$
\rho_{W 0}=\operatorname{Cexp}\left\{-\frac{1}{2 \hbar} \sum_{j=1}^{D-2} \sum_{n \neq 0} \frac{1}{|n|}\left(\left(P_{n}^{j}\right)^{2}+4 n^{2}\left(Q_{n}^{j}\right)^{2}\right)\right\} \delta\left(p^{1}\right) \ldots \delta\left(p^{D-2}\right) \delta\left(p^{+}\right) .
$$

Observe that $\rho_{W 0}$ is defined by Eqs. (3.29), (3.27) and (3.28) uniquely up to an arbitrary real constant factor $C>0$. This fact can be interpreted in deformation quantization formalism as the uniqueness of the vacuum state.

Then any higher state can be obtained as an appropriate product of the form

$$
\begin{gathered}
\left(a_{n_{1}}^{* i_{1}} \ldots a_{n_{s}}^{* i_{s}}\right) *\left\{C \exp \left(-\frac{1}{2 \hbar} \sum_{j=1}^{D-2} \sum_{n \neq 0} \frac{1}{|n|}\left(\left(P_{n}^{j}\right)^{2}+4 n^{2}\left(Q_{n}^{j}\right)^{2}\right)\right)\right. \\
\left.\delta\left(p^{1}-p_{0}^{1}\right) \ldots \delta\left(p^{D-2}-p_{0}^{D-2}\right) \delta\left(p^{+}-p_{0}^{+}\right)\right\} *\left(a_{n_{s}}^{i_{s}} \ldots a_{n_{1}}^{i_{1}}\right),
\end{gathered}
$$

(compare with [18]).

An interesting question is when a real function $\rho_{W}\left(x^{-}, Q, p^{+}, P\right)$ satisfying Eqs. (3.27) and (3.28) is the Wigner function of a pure state. The answer to this question in the case 
of a system of particles can be found in a beautiful paper by Tataskii [26]. In our case the solution is quite similar. To this end denote

$\gamma\left(x^{-}, Q, \widetilde{x}^{-}, \widetilde{Q}\right):=\int d p^{+} d P \rho_{W}\left(\frac{x^{-}+\widetilde{x}^{-}}{2}, \frac{Q+\widetilde{Q}}{2}, p^{+}, P\right) \exp \left\{\frac{i}{\hbar}\left[-\left(x^{-}-\widetilde{x}^{-}\right) p^{+}+(Q-\widetilde{Q}) P\right]\right\}$.

From Eq. (3.25) it follows that if $\rho_{W}$ is the Wigner function of the pure state $|\Psi\rangle\langle\Psi|$ then

$$
\begin{gathered}
\frac{\partial^{2} \ln \gamma\left(x^{-}, Q, \widetilde{x}^{-}, \widetilde{Q}\right)}{\partial x^{-} \partial \widetilde{x}^{-}}=\frac{\partial^{2} \ln \gamma\left(x^{-}, Q, \widetilde{x}^{-}, \widetilde{Q}\right)}{\partial x^{-} \partial \widetilde{Q}_{n}^{j}} \\
=\frac{\partial^{2} \ln \gamma\left(x^{-}, Q, \widetilde{x}^{-}, \widetilde{Q}\right)}{\partial Q_{n}^{j} \partial \widetilde{x}^{-}}=\frac{\partial^{2} \ln \gamma\left(x^{-}, Q, \widetilde{x}^{-}, \widetilde{Q}\right)}{\partial Q_{m}^{j} \partial \widetilde{Q}_{n}^{k}}=0
\end{gathered}
$$

for every $j, k=1, \ldots, D-2$ and $m, n \in \mathbb{Z}$ (we put $x^{i} \equiv Q_{0}^{j}, p^{j} \equiv P_{0}^{j}$ ).

Conversely, let $\gamma$ satisfies Eq. (3.35). The general solution of (3.35) reads

$$
\gamma\left(x^{-}, Q, \widetilde{x}^{-}, \widetilde{Q}\right)=\Psi_{1}\left(x^{-}, Q\right) \Psi_{2}\left(\widetilde{x}^{-}, \widetilde{Q}\right) .
$$

As the function $\rho_{W}$ is assumed to be real we get from Eq. (3.34)

$$
\gamma^{*}\left(x^{-}, Q, \widetilde{x}^{-}, \widetilde{Q}\right)=\gamma\left(\widetilde{x}^{-}, \widetilde{Q}, x^{-}, Q\right) .
$$

Consequently, Eq. (3.36) has the form

$$
\gamma\left(x^{-}, Q, \widetilde{x}^{-}, \widetilde{Q}\right)=A \Psi_{1}\left(x^{-}, Q\right) \Psi_{1}^{*}\left(\widetilde{x}^{-}, \widetilde{Q}\right),
$$

where, by the assumption (3.28), $A$ is a positive real constant. Finally, defining $\Psi:=$ $\sqrt{A} \Psi_{1}\left(x^{-}, Q\right)$ one obtains

$$
\gamma\left(x^{-}, Q, \widetilde{x}^{-}, \widetilde{Q}\right)=\Psi\left(x^{-}, Q\right) \Psi^{*}\left(\widetilde{x}^{-}, \widetilde{Q}\right) .
$$

Substituting $x^{-} \mapsto x^{-}+\frac{\xi^{-}}{2}, Q \mapsto Q+\frac{\xi}{2}, \widetilde{x}^{-} \mapsto x^{-}-\frac{\xi^{-}}{2}, \widetilde{Q} \mapsto Q-\frac{\xi^{-}}{2}$, multiplying both sides by $\exp \left\{-\frac{i}{\hbar}\left(-\xi^{-} p^{+}+\xi \cdot P\right)\right\}$ and integrating with respect to $d\left(\frac{\xi^{-}}{2 \pi \hbar}\right) d\left(\frac{\xi}{2 \pi \hbar}\right)$ we get exactly the relation (3.21). This means that our function $\rho_{W}$ is the Wigner function of the pure state $\Psi\left(x^{-}, Q\right)$. Thus we arrive at the following 


\section{Theorem 2.1}

A real function $\rho_{W}\left(x^{-}, Q, p^{+}, P\right)$ satisfying also the conditions (3.27) and (3.28) is the Wigner function of some pure state if and only if the function $\gamma\left(x^{-}, Q, \widetilde{x}, \widetilde{Q}\right)$ defined by (3.34) satisfies Eqs. (3.35)

In terms of $\left(x^{-}, X, p^{+}, \Pi\right)$ variables the conditions (3.35) read

$$
\begin{gathered}
\frac{\partial^{2} \ln \gamma\left[x^{-}, X, \widetilde{x}^{-}, \widetilde{X}\right]}{\partial x^{-} \partial \widetilde{x}^{-}}=\frac{\partial}{\partial x^{-}} \frac{\delta \ln \gamma\left[x^{-}, X, \widetilde{x}^{-}, \tilde{X}\right]}{\delta \widetilde{X}} \\
=\frac{\partial}{\partial \widetilde{x}^{-}} \frac{\delta \ln \gamma\left[x^{-}, X, \widetilde{x}^{-}, \widetilde{X}\right]}{\delta X}=\frac{\delta^{2} \ln \gamma\left[x^{-}, X, \widetilde{x}^{-}, \widetilde{X}\right]}{\delta X \delta \widetilde{X}}=0 .
\end{gathered}
$$

\subsection{Open Strings}

This is a simple matter to carry over the results of the preceding subsection to the case of open string. The thing we must take in care is that the subindice $n$ standing at $Q_{n}^{j}$, $P_{n}^{j}, a_{n}^{j}$ and $a_{n}^{* j}$ takes the values $n=1, \ldots, \infty$. (We let also $n$ be zero in the formulas where $\left.Q_{0}^{j} \equiv x^{j}, P_{0}^{j} \equiv p^{j}\right)$. Moreover, we should remember that now the oscillator frequencies $\omega_{n}=n \in \mathbb{Z}_{+}$, and not $2|n|$, as before, and also that $\widetilde{\alpha}_{n}, \widetilde{\alpha}_{-n}$ don't appear. Thus in the present case one gets

$$
\begin{gathered}
*=\exp \left\{\frac{i \hbar}{2} \overleftrightarrow{\mathcal{P}}\right\} \\
=\exp \left\{\frac{i \hbar}{2}\left[\left(\frac{\overleftarrow{\partial}}{\partial p^{+}} \frac{\vec{\partial}}{\partial x^{-}}-\frac{\overleftarrow{\partial}}{\partial x^{-}} \frac{\vec{\partial}}{\partial p^{+}}\right)+\sum_{j=1}^{D-2}\left(\frac{\overleftarrow{\partial}}{\partial x^{j}} \frac{\vec{\partial}}{\partial p^{j}}-\frac{\overleftarrow{\partial}}{\partial p^{j}} \frac{\vec{\partial}}{\partial x^{j}}\right)\right]\right\} \\
\exp \left\{\frac{1}{2} \sum_{j=1}^{D-2} \sum_{n=1}^{\infty}\left(\frac{\overleftarrow{\partial}}{\partial a_{n}^{j}} \frac{\vec{\partial}}{\partial a_{n}^{* j}}-\frac{\overleftarrow{\partial}}{\partial a_{n}^{* j}} \frac{\vec{\partial}}{\partial a_{n}^{j}}\right)\right\} \\
=\ldots \exp \left\{\frac{\hbar}{2} \sum_{j=1}^{D-2} \sum_{n \neq 0} n \frac{\overleftarrow{\partial}}{\partial \alpha_{n}^{j}} \frac{\vec{\partial}}{\partial \alpha_{-n}^{j}}\right\} .
\end{gathered}
$$

Then the Wigner function of the ground state reads now 


$$
\begin{gathered}
\rho_{W 0}=C \exp \left\{-2 \sum_{j=1}^{D-2} \sum_{n=1}^{\infty} a_{n}^{j} a_{n}^{* j}\right\} \delta\left(p^{1}\right) \ldots \delta\left(p^{D-2}\right) \delta\left(p^{+}\right) \\
=C \exp \left\{-\frac{1}{\hbar} \sum_{j=1}^{D-2} \sum_{n=1}^{\infty} \frac{1}{n}\left(\left(P_{n}^{j}\right)^{2}+n^{2}\left(Q_{n}^{j}\right)^{2}\right)\right\} \delta\left(p^{1}\right) \ldots \delta\left(p^{D-2}\right) \delta\left(p^{+}\right),
\end{gathered}
$$

with $C>0$. (Compare with [18] Eq. (3.21)).

\section{Hamiltonian, Square Mass, Normal Ordering and Virasoro Algebra}

To proceed further with deformation quantization of bosonic string we must take into account that not only the Weyl ordering but also the normal ordering should be implemented into this quantization. To this end we first consider the Casimir effect in string theory. Consider the real scalar field $\Phi(\tau, \sigma)$ on the cylindrical space time $\mathbb{R} \times \mathbf{S}^{1}$. The circunference of $\mathbf{S}^{1}$ is $L$. The standard expansion of $\Phi(\tau, \sigma)$ satisfying the boundary conditions $\Phi(\tau, \sigma)=\Phi(\tau, \sigma+n L)$ for all $n \in \mathbb{Z}$ reads (compare with Eq. (2.12))

$\Phi(\tau, \sigma)=x+\frac{1}{L} p \tau+\frac{1}{\sqrt{L}} \sum_{n \neq 0} \sqrt{\frac{\hbar}{2 \omega_{n}}}\left\{a_{n} \exp \left(i\left(\frac{2 \pi n}{L} \sigma-\omega_{n} \tau\right)\right)+a_{n}^{*} \exp \left(-i\left(\frac{2 \pi n}{L} \sigma-\omega_{n} \tau\right)\right)\right\}$

where $\omega_{n}=\frac{2 \pi|n|}{L}$. The conjugate momentum $\Pi(\tau, \sigma)=\dot{\Phi}(\tau, \sigma)$. Employing the deformation quantization formalism one can show [18] that the expected value of the energy density $\left\langle T_{00}\right\rangle(L)$ of the ground state is (the Casimir effect)

$$
\left\langle T_{00}\right\rangle(L)=-\frac{\pi \hbar}{6 L^{2}} .
$$

(In terms of the usual quantum field theory see [32-34].)

Consequently, for the total energy $E_{0}(L)$ of the ground state one gets

$$
E_{0}(L)=L \cdot\left\langle T_{00}\right\rangle(L)=-\frac{\pi \hbar}{6 L}
$$


Consider now the real scalar field $\Phi(\tau, \sigma)$ on $\mathbb{R} \times[0, L]$ but with the boundary conditions $\frac{\partial \Phi(\tau, 0)}{\partial \sigma}=0=\frac{\partial \Phi(\tau, L)}{\partial \sigma}$ for all $\tau \in \mathbb{R}$. It is a simple matter to show that now the expansion of $\Phi(\tau, \sigma)$ takes the following form

$\Phi(\tau, \sigma)=x+\frac{1}{L} p \tau+\frac{1}{\sqrt{2 L}} \sum_{n \neq 0} \sqrt{\frac{\hbar}{2 \omega_{n}}}\left\{a_{n} \exp \left(i\left(\frac{\pi n}{L} \sigma-\omega_{n} \tau\right)\right)+a_{n}^{*} \exp \left(-i\left(\frac{\pi n}{L} \sigma-\omega_{n} \tau\right)\right)\right\}$

where $\omega_{n}=\frac{\pi|n|}{L}$ and $a_{n}=a_{-n}$. Comparing (4.1) with (4.4) one quickly arrives at the conclusion that the oscillating part in (4.4) is mutatis mutandi the same as in (4.1) if in (4.1) $L$ is changed to $2 L$. Hence it follows that in the present case the Casimir effect can be obtained from (4.2) by inserting $2 L$ instead of $L$. Thus we have now

$$
\left\langle T_{00}\right\rangle(L)=-\frac{\pi \hbar}{24 L^{2}}
$$

and for the total energy of the ground state

$$
E_{0}(L)=L \cdot\left\langle T_{00}\right\rangle(L)=-\frac{\pi \hbar}{24 L} .
$$

We use the above results to the deformation quantization of bosonic strings.

\subsection{Closed Strings}

In this case one can consider $X^{j}(\tau, \sigma), j=1, \ldots, D-2$, to be $D-2$ real scalar massless fields on the cylindrical worldsheet $\mathbb{R} \times \mathbf{S}^{1}$ with $L=\pi$. Therefore, by Eq. (4.3), the vacuum energy $E_{0}$ reads now

$$
E_{0}=-\frac{\hbar(D-2)}{6}=:-4 a .
$$

To obtain this $E_{0}$ from the eigenvalue equation we put $\hat{\mathcal{N}}^{\prime} H$

$$
\hat{\mathcal{N}}^{\prime}:=\exp \left\{\sum_{j=1}^{D-2} \sum_{n \neq 0}\left(-\frac{1}{2}+\gamma_{n}\right) \frac{\partial^{2}}{\partial a_{n}^{j} \partial a_{n}^{* j}}\right\}
$$




$$
=\exp \left\{\hbar \sum_{j=1}^{D-2} \sum_{n=1}^{\infty} n\left(\left(-\frac{1}{2}+\gamma_{n}\right) \frac{\partial^{2}}{\partial \alpha_{n}^{j} \partial \alpha_{-n}^{j}}+\left(-\frac{1}{2}+\gamma_{-n}\right) \frac{\partial^{2}}{\partial \widetilde{\alpha}_{n}^{j} \partial \widetilde{\alpha}_{-n}^{j}}\right)\right\}
$$

instead of the Hamiltonian $H$ given by Eq. (2.34). Then

$$
\hat{\mathcal{N}}^{\prime} H * \rho_{W 0}=-4 a \rho_{W 0}
$$

if

$$
\sum_{n \neq 0}|n| \gamma_{n}=-\frac{D-2}{12}
$$

From the previous work [18] we know that the Weyl image of $\hat{\mathcal{N}}^{\prime} H$ reads

$$
W\left(\hat{\mathcal{N}}^{\prime} H\right)=: W(H):-4 a,
$$

where : $W(H)$ : is the normal ordered operator $W(H)$ and it can be written as follows

$$
\begin{gathered}
: W(H):=W(\hat{\mathcal{N}} H) \\
\hat{\mathcal{N}}:=\exp \left\{-\frac{1}{2} \sum_{j=1}^{D-2} \sum_{n \neq 0} \frac{\partial^{2}}{\partial a_{n}^{j} \partial a_{n}^{* j}}\right\}=\exp \left\{-\frac{\hbar}{2} \sum_{j=1}^{D-2} \sum_{n=1}^{\infty} n\left(\frac{\partial^{2}}{\partial \alpha_{n}^{j} \partial \alpha_{-n}^{j}}+\frac{\partial^{2}}{\partial \widetilde{\alpha}_{n}^{j} \partial \widetilde{\alpha}_{-n}^{j}}\right)\right\} .
\end{gathered}
$$

Analogously for the square mass given by Eq. (2.32) we get

$$
\hat{\mathcal{N}}^{\prime} M^{2} * \rho_{W 0}=-8 \pi T a \rho_{W 0} .
$$

One quickly finds that

$$
W\left(\hat{\mathcal{N}}^{\prime} M^{2}\right)=W\left(\hat{\mathcal{N}} M^{2}\right)-8 \pi T a=: W\left(M^{2}\right):-8 \pi T a .
$$

Given the normal ordering operator $\hat{\mathcal{N}}$ and the generalized normal ordering operator $\hat{\mathcal{N}}^{\prime}$ one can define new star products which are cohomologically equivalent to the Moyal 
*-product (see Eqs. $(3.13)$ or $(3.18)$ ). These star products will be denoted by $* \mathcal{N}$ and $*_{\mathcal{N}^{\prime}}$ respectively and they are given by

$$
\begin{gathered}
F_{1} * \mathcal{N} F_{2}=\hat{\mathcal{N}}^{-1}\left(\hat{\mathcal{N}} F_{1} * \hat{\mathcal{N}} F_{2}\right) \\
F_{1} * \mathcal{N}^{\prime} F_{2}=\hat{\mathcal{N}}^{\prime-1}\left(\hat{\mathcal{N}}^{\prime} F_{1} * \hat{\mathcal{N}}^{\prime} F_{2}\right) .
\end{gathered}
$$

Consequently the eigenvalue equations for the Hamiltonian or the square mass read (compare with Eq. (4.9) or (4.13))

$$
\begin{gathered}
H * \mathcal{N}^{\prime} \rho_{W}^{\mathcal{N}^{\prime}}=E \rho_{W}^{\mathcal{N}^{\prime}} \Longrightarrow H *_{\mathcal{N}^{\prime}} \rho_{W 0}^{\mathcal{N}^{\prime}}=-4 a \rho_{W 0}^{\mathcal{N}^{\prime}} \\
M^{2} *_{\mathcal{N}^{\prime}} \rho_{W}^{\mathcal{N}^{\prime}}=\mu^{2} \rho_{W}^{\mathcal{N}^{\prime}} \Longrightarrow M^{2} *_{\mathcal{N}^{\prime}} \rho_{W 0}^{\mathcal{N}^{\prime}}=-8 \pi T a \rho_{W 0}^{\mathcal{N}^{\prime}}
\end{gathered}
$$

where $\rho_{W}^{\mathcal{N}^{\prime}}:=\hat{\mathcal{N}}^{-1} \rho_{W}$.

It is an easy matter to show that

$$
\begin{array}{ll}
\alpha_{-n} *_{\mathcal{N}} \alpha_{n}=\alpha_{-n} \alpha_{n}, & \alpha_{n} *_{\mathcal{N}} \alpha_{-n}=\alpha_{n} \alpha_{-n}+\hbar n \\
\widetilde{\alpha}_{-n} *_{\mathcal{N}} \widetilde{\alpha}_{n}=\widetilde{\alpha}_{-n} \widetilde{\alpha}_{n}, \quad \widetilde{\alpha}_{n} *_{\mathcal{N}} \widetilde{\alpha}_{-n}=\widetilde{\alpha}_{n} \widetilde{\alpha}_{-n}+\hbar n
\end{array}
$$

for all $n \in \mathbb{Z}_{+}$. All other products are the usual products.

Taking into account Eq. (4.17) one gets

$$
\begin{array}{r}
\left\{\alpha_{m}^{-}, \alpha_{n}^{-}\right\}^{(\mathcal{N})}=-i \frac{2 \sqrt{\pi T}}{p^{+}}(m-n) \hat{\mathcal{N}} \alpha_{m+n}^{-}-i \hbar \frac{4 \pi T}{\left(p^{+}\right)^{2}} \frac{D-2}{12} m\left(m^{2}-1\right) \delta_{m+n, 0} \\
\left\{\widetilde{\alpha}_{m}^{-}, \widetilde{\alpha}_{n}^{-}\right\}^{(\mathcal{N})}=-i \frac{2 \sqrt{\pi T}}{p^{+}}(m-n) \hat{\mathcal{N}} \widetilde{\alpha}_{m+n}^{-}-i \hbar \frac{4 \pi T}{\left(p^{+}\right)^{2}} \frac{D-2}{12} m\left(m^{2}-1\right) \delta_{m+n, 0},
\end{array}
$$

where $\left\{\alpha_{m}^{-}, \alpha_{n}^{-}\right\}^{(\mathcal{N})}:=\frac{1}{i \hbar}\left(\alpha_{m}^{-} *_{\mathcal{N}} \alpha_{n}^{-}-\alpha_{n}^{-} *_{\mathcal{N}} \alpha_{m}^{-}\right)$etc. Thus we arrive at the Virasoro algebra with a central extension. 
[Remark: Calculations in Eq. (4.18) are rather formal. To perform them one must always put $\alpha_{n}$ on the right to $\alpha_{-m} m, n \in \mathbb{Z}_{+}, m \neq n$, and the same for $\widetilde{\alpha}_{n}$ and $\widetilde{\alpha}_{-m}$. See the analogous calculations in terms of operator language [4].]

\subsection{Open Strings}

Here we can find the energy of the vacuum state $E_{0}$ by substituting $L=\pi$ into (4.6) and taking into account that we deal with $D-2$ scalar fields. Hence

$$
E_{0}=-\frac{\hbar(D-2)}{24}=-a .
$$

Now the normal ordering operator $\hat{\mathcal{N}}$ and the generalized normal ordering operator $\hat{\mathcal{N}}^{\prime}$ read

$$
\begin{gathered}
\hat{\mathcal{N}}=\exp \left\{-\frac{1}{2} \sum_{j=1}^{D-2} \sum_{n=1}^{\infty} \frac{\partial^{2}}{\partial a_{n}^{j} \partial a_{n}^{* j}}\right\}=\exp \left\{-\frac{\hbar}{2} \sum_{j=1}^{D-2} \sum_{n=1}^{\infty} n \frac{\partial^{2}}{\partial \alpha_{n}^{j} \partial \alpha_{-n}^{j}}\right\} \\
\hat{\mathcal{N}}^{\prime}==\exp \left\{\sum_{j=1}^{D-2} \sum_{n=1}^{\infty}\left(\left(-\frac{1}{2}+\beta_{n}\right) \frac{\partial^{2}}{\partial a_{n}^{j} \partial a_{n}^{* j}}\right)\right\}=\exp \left\{\hbar \sum_{j=1}^{D-2} \sum_{n=1}^{\infty} n\left(\left(-\frac{1}{2}+\beta_{n}\right) \frac{\partial^{2}}{\partial \alpha_{n}^{j} \partial \alpha_{-n}^{j}}\right)\right\}
\end{gathered}
$$

where

$$
\sum_{n=1}^{\infty} n \beta_{n}=-\frac{D-2}{24}
$$

Then

$$
\begin{gathered}
H *_{\mathcal{N}^{\prime}} \rho_{W}^{\mathcal{N}^{\prime}}=E \rho_{W}^{\mathcal{N}^{\prime}} \Longrightarrow H *_{\mathcal{N}^{\prime}} \rho_{W 0}^{\mathcal{N}^{\prime}}=-a \rho_{W 0}^{\mathcal{N}^{\prime}} \\
M^{2} *_{\mathcal{N}^{\prime}} \rho_{W}^{\mathcal{N}^{\prime}}=\mu^{2} \rho_{W}^{\mathcal{N}^{\prime}} \Longrightarrow M^{2} *_{\mathcal{N}^{\prime}} \rho_{W 0}^{\mathcal{N}^{\prime}}=-2 \pi T a \rho_{W 0}^{\mathcal{N}^{\prime}} .
\end{gathered}
$$

Thus, as before, the ground state is the tachyonic one.

Finally, the Virasoro algebra with a central extension reads now 


$$
\left\{\alpha_{m}^{-}, \alpha_{n}^{-}\right\}^{(\mathcal{N})}=-i \frac{\sqrt{\pi T}}{p^{+}}(m-n) \hat{\mathcal{N}} \alpha_{m+n}^{-}-i \hbar \frac{\pi T}{\left(p^{+}\right)^{2}} \frac{D-2}{12} m\left(m^{2}-1\right) \delta_{m+n, 0} .
$$

\section{Some Simple Example: The Wightman Functions}

Here we are going to present a simple example of calculations within the deformation

quantization formalism. Namely, we find the Wightman (Green) functions $\left\langle X^{j}(\tau, \sigma) *\right.$ $\left.X^{k}\left(\tau^{\prime}, \sigma^{\prime}\right)\right\rangle$. By the definition (see(3.23))

$$
\left\langle X^{j}(\tau, \sigma) * X^{k}\left(\tau^{\prime}, \sigma^{\prime}\right)\right\rangle=\frac{\int d x^{-} d p^{+} d Q d P \rho_{W 0}\left(x^{-}, Q, p^{+}, P\right) X^{j}(\tau, \sigma) * X^{k}\left(\tau^{\prime}, \sigma^{\prime}\right)}{\int d x^{-} d p^{+} d Q d P \rho_{W 0}\left(x^{-}, Q, p^{+}, P\right)} .
$$

where $\rho_{W 0}$ is given by Eq. (3.32) (closed string) or Eq. (3.42) (open string) and $X^{j}(\tau, \sigma)$ is given by Eq. (2.41) (closed string) or by Eq. (2.56) (open string).

\subsection{Closed Strings}

Employing Eqs. (2.42) and (3.18) and perfoming simple integrations (Gaussian integrals) one gets

$$
\left\langle Q_{m}^{j}(\tau) * Q_{n}^{k}\left(\tau^{\prime}\right)\right\rangle=\delta_{j k} \delta_{m n} \frac{\hbar}{4|m|} \exp \left(-2 i|m|\left(\tau-\tau^{\prime}\right)\right), \quad m, n \neq 0 .
$$

Hence, differentiating (5.2) with respect to $\tau$ or/and $\tau^{\prime}$ we obtain

$$
\begin{gathered}
\left\langle Q_{m}^{j}(\tau) * P_{n}^{k}\left(\tau^{\prime}\right)\right\rangle=\delta_{j k} \delta_{m n} \frac{i \hbar}{2} \exp \left(-2 i|m|\left(\tau-\tau^{\prime}\right)\right)=-\left\langle P_{m}^{j}(\tau) * Q_{n}^{k}\left(\tau^{\prime}\right)\right\rangle \\
\left\langle P_{m}^{j}(\tau) * P_{n}^{k}\left(\tau^{\prime}\right)\right\rangle=\delta_{j k} \delta_{m n} \hbar|m| \exp \left(-2 i|m|\left(\tau-\tau^{\prime}\right)\right), \quad m, n \neq 0 .
\end{gathered}
$$


Then we have also

$$
\begin{gathered}
\left\langle x^{j} * p^{k}\right\rangle=\delta_{j k} \frac{i \hbar}{2}=-\left\langle p^{j} * x^{k}\right\rangle \\
\left\langle p^{j} * p^{k}\right\rangle=0, \quad\left\langle x^{j} * x^{k}\right\rangle=\delta_{j k}\left\langle\left(x^{j}\right)^{2}\right\rangle .
\end{gathered}
$$

Using Eqs. (5.2), (5.3) and (5.4) one easily finds

$\left\langle X^{j}(\tau, \sigma) * X^{k}\left(\tau^{\prime}, \sigma^{\prime}\right)\right\rangle=\delta_{j k}\left\{\left\langle x^{j} x^{k}\right\rangle+\frac{i \hbar}{2 \pi T}\left(\tau^{\prime}-\tau\right)+\frac{\hbar}{4 \pi T} \sum_{n \neq 0} \frac{\exp \left(2 i\left[n\left(\sigma-\sigma^{\prime}\right)-|n|\left(\tau-\tau^{\prime}\right)\right]\right)}{|n|}\right\}$.

Performing the summations in Eq. (5.5) and removing the part independent of the coordinates $\left(\tau, \sigma, \tau^{\prime}, \sigma^{\prime}\right)$ we have

$$
\left\langle X^{j}(\tau, \sigma) * X^{k}\left(\tau^{\prime}, \sigma^{\prime}\right)\right\rangle \sim \delta_{j k}\left(-\frac{\hbar}{4 \pi T}\right)\left\{\ln \left|\sin \left[\tau^{\prime}-\sigma^{\prime}-(\tau-\sigma)\right]\right|+\ln \left|\sin \left[\tau^{\prime}+\sigma^{\prime}-(\tau+\sigma)\right]\right|\right\} .
$$

(Compare with $[1,2,4,33])$.

\subsection{Open Strings}

For open strings we get

$$
\begin{gathered}
\left\langle Q_{m}^{j}(\tau) * Q_{n}^{k}\left(\tau^{\prime}\right)\right\rangle=\delta_{j k} \delta_{m n} \frac{\hbar}{2|m|} \exp \left(-i|m|\left(\tau-\tau^{\prime}\right)\right), \\
\left\langle Q_{m}^{j}(\tau) * P_{n}^{k}\left(\tau^{\prime}\right)\right\rangle=\delta_{j k} \delta_{m n} \frac{i \hbar}{2} \exp \left(-i|m|\left(\tau-\tau^{\prime}\right)\right)=-\left\langle P_{m}^{j}(\tau) * Q_{n}^{k}\left(\tau^{\prime}\right)\right\rangle, \\
\left\langle P_{m}^{j}(\tau) * P_{n}^{k}\left(\tau^{\prime}\right)\right\rangle=\delta_{j k} \delta_{m n} \frac{\hbar|m|}{2} \exp \left(-i|m|\left(\tau-\tau^{\prime}\right)\right), \quad m, n \neq 0 .
\end{gathered}
$$

Then 
$\left\langle X^{j}(\tau, \sigma) * X^{k}\left(\tau^{\prime}, \sigma^{\prime}\right)\right\rangle=\delta_{j k}\left\{\left\langle x^{j} x^{k}\right\rangle+\frac{i \hbar}{2 \pi T}\left(\tau^{\prime}-\tau\right)+\frac{\hbar}{\pi T} \sum_{n=1}^{\infty} \frac{\exp \left(-i n\left(\tau-\tau^{\prime}\right)\right) \cos (n \sigma) \cos \left(n \sigma^{\prime}\right)}{n}\right\}$

Performing the summations in Eq. (5.8) and removing the part independent of the coordinates we get

$$
\begin{gathered}
\left\langle X^{j}(\tau, \sigma) * X^{k}\left(\tau^{\prime}, \sigma^{\prime}\right)\right\rangle \sim \delta_{j k}\left(-\frac{\hbar}{4 \pi T}\right)\left\{\ln \left|\sin \left(\frac{\tau^{\prime}-\sigma^{\prime}-(\tau-\sigma)}{2}\right)\right|+\ln \left|\sin \left(\frac{\tau^{\prime}-\sigma^{\prime}-(\tau+\sigma)}{2}\right)\right|\right. \\
\left.\quad+\ln \left|\sin \left(\frac{\tau^{\prime}+\sigma^{\prime}-(\tau-\sigma)}{2}\right)\right|+\ln \left|\sin \left(\frac{\tau^{\prime}+\sigma^{\prime}-(\tau+\sigma)}{2}\right)\right|\right\}
\end{gathered}
$$

\section{Final Remarks}

In this paper we have investigated bosonic string theory within the deformation quantization formalism. As it is seen deformation quantization provides us with a tool which enables one to describe quantum bosonic string in terms of deformed Poisson-Lie algebra.

The most intriguing problem is to obtain the similar result for the case of superstring theory. Some works on defomation quantization of supersymmetric field theory (see e.g. $[35,36,37])$ seem to be crucial in searching for a solution for the problem. Another interesting possibility is the extension of the matters considerd here, to the case of interacting strings and interacting superstrings. We are going to consider these problems in the near future.

\section{Acknowledgements}

This paper was partially supported by CINVESTAV and CONACyT (México) and by KBN (Poland). 
One of us (M.P.) is indebted to the staff of Departmento de Física at CINVESTAV (México) for warm hospitality. 


\section{References}

[1] M.B. Green, J.H. Schwarz and E. Witten, Superstring Theory, Two volumes, Cambridge Univesity Press, Cambridge (1986).

[2] J. Polchinski, String Theory, Two volumes, Cambridge University Press, Cambridge (1998).

[3] B. Hatfield, Quantum Field Theory of Point Particles and Stings, Addison-Wesley Publishing, Redwood City, (1992).

[4] D. Lüst and S. Theisen, Lectures on String Theory, Lecture Notes in Physics bf 346, Springer-Verlag, Berlin (1989).

[5] S.T. Yau (ed.), Mathematical Aspects of String Theory, World Scientific, Singapore (1987).

[6] S.T. Yau (ed.), Mirror Symmetry I, American Mathematical Society, Providence USA (1998); B. Greene and S.T. Yau (eds.), Mirror Symmetry II, American Mathematical Society, Providence USA (1997).

[7] C. Vafa, "Geometric Physics", hep-th/9810149.

[8] E. Witten, "D-branes and K-Theory", JHEP 812:019 (1998); hep-th/9810188.

[9] A. Connes, M. Douglas and A. Schwarz, 'Noncommutative Geometry and Matrix Theory: Compactification on Tori", JHEP 9802:003 (1998), hep-th/9711162.

[10] V. Schomerus, "D-branes and Deformation Quantization", JHEP 9906:030 (1999).

[11] N. Seiberg and E. Witten, "String Theory and Noncommutative Geometry", JHEP 9909:03 (1999), hep-th/9908142.

[12] J. Dito, Lett. Math. Phys. 20 (1990) 125; Lett. Math. Phys. 27 (1993) 73.

[13] F. Antonsen, Phys. Rev. D 56, 920 (1997).

[14] F. Antonsen, "Deformation Quantization of Constrained Systems", gr-qc/9710021.

[15] F. Antonsen, "Deformation Quantization of Gravity", gr-qc/9712012.

[16] T. Curtright D. Fairlie and C. Zachos, Phys. Rev. D 58, 025002 (1998).

[17] T. Curtright and C. Zachos, J. Phys. A 32, 771 (1999).

[18] H. García-Compeán, J.F. Plebański, M. Przanowski and F.J. Turrubiates, "Deformation Quantization of Classical Fields", hep-th/9909206.

[19] V.B. Berestetskii, E.M. Lifshitz and L.P. Pitaevskii, Relativistic Quantum Theory, (Pergamon Press, New York, 1971).

[20] H. Weyl, Group Theory and Quantum Mechanics, (Dover, New York, 1931).

[21] E.P. Wigner, Phys. Rev. 40, 749 (1932).

[22] J.E. Moyal, Proc. Camb. Phil. Soc. 45, 99 (1949).

[23] R.L. Stratonovich, Sov. Phys. JETP 31 (1956) 1012.

[24] G.S. Agarwal and E. Wolf, Phys. Rev. D 2 (1970) 2161; 2206.

[25] F. Bayen, M. Flato, C. Fronsdal, A. Lichnerowicz and D. Sternheimer, Ann. Phys. 111, 61 (1978); Ann. Phys. 111, 111 (1978). 
[26] W.I. Tatarskii, Usp. Fiz. Nauk 139 (1983) 587.

[27] M. Hillery, R.F. O'Connell, M.O. Scully and E.P. Wigner, Phys. Rep. 106, 121 (1984).

[28] J.F. Cariñena, J.M. Gracia Bondía and J.C. Varilly, J. Phys. A: Math. Gen. 23, 901 (1990).

[29] M. Gadella, Fortschr. Phys. 43 (1995) 229.

[30] J.F. Plebański, M. Przanowski and J. Tosiek, Acta Phys. Pol. B27 1961 (1996).

[31] M. Bordemann, N. Neumaier and S. Waldman, J. Geom. Phys. 29 (1999) 199.

[32] B.S. Kay, Phys. Rev. D20, 3052 (1979).

[33] N.D. Birrell and P.C.W. Davis, Quantum Fields in Curved Space (Cambridge University Press, Cambridge, 1982).

[34] C.J. Isham, Proc. R. Soc. Lond. A362, 383 (1978).

[35] I.A. Batalin and E.S. Fradkin, Ann. Inst. Henri Poincaré, 49, 145 (1988).

[36] S. Ferrara and M.A. Lledó, "Some Aspects of Deformation of Supersymmetric Field Theories", hep-th/0002084.

[37] S. Terashima, "A Note on Superfields and Noncommutative Geometry", hep-th/0002119. 